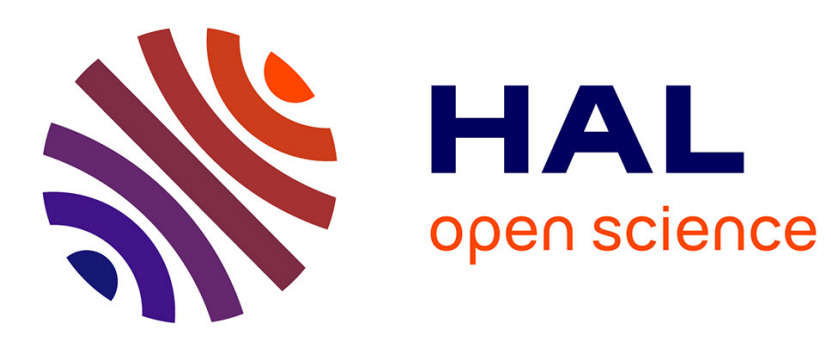

\title{
Adaptive Attitude Tracking Control of Rigid Body Systems with Unknown Inertia and Gyro-bias
}

Abdelaziz Benallegue, Yacine Chitour, Abdelhamid Tayebi

\section{To cite this version:}

Abdelaziz Benallegue, Yacine Chitour, Abdelhamid Tayebi. Adaptive Attitude Tracking Control of Rigid Body Systems with Unknown Inertia and Gyro-bias. IEEE Transactions on Automatic Control, 2018, 63 (11), pp.3986-3993. 10.1109/tac.2018.2808443 . hal-01507341v2

\section{HAL Id: hal-01507341 \\ https://hal.science/hal-01507341v2}

Submitted on 14 Feb 2018

HAL is a multi-disciplinary open access archive for the deposit and dissemination of scientific research documents, whether they are published or not. The documents may come from teaching and research institutions in France or abroad, or from public or private research centers.
L'archive ouverte pluridisciplinaire HAL, est destinée au dépôt et à la diffusion de documents scientifiques de niveau recherche, publiés ou non, émanant des établissements d'enseignement et de recherche français ou étrangers, des laboratoires publics ou privés. 


\title{
Adaptive Attitude Tracking Control of Rigid Body Systems with Unknown Inertia and Gyro-bias
}

\author{
Abdelaziz Benallegue, Yacine Chitour, Abdelhamid Tayebi
}

\begin{abstract}
This note presents a new adaptive attitude tracking controller for rigid body systems, with unknown inertia and unknown gyrobias, using inertial vector measurements. The proposed control scheme guarantees almost global asymptotic convergence of the attitude and angular velocity to their desired values. Simulation results are provided to illustrate the effectiveness of the proposed approach.
\end{abstract}

\section{INTRODUCTION}

The rigid body attitude tracking problem is still relevant, despite having been extensively studied in the literature for several decades. Several solutions have been proposed in the literature in the full state measurement case (i.e., attitude and angular velocity available for feedback) using different attitude representations, see for instance, [1]-[3]. Since there is no sensor that directly measures the orientation, the explicit use of the attitude in the control law calls for efficient attitude estimation algorithms (observers) that reconstruct the attitude from the measurements provided by some appropriate sensors, such as inertial measurements units (IMUs) typically including a gyroscope, an accelerometer and a magnetometer. The attitude can be determined using either static reconstruction algorithms [4] which are vulnerable to measurement noise, or dynamic attitude estimation algorithms such as Kalman-type filters [5] and nonlinearcomplimentary filters [6]. The attitude tracking problem with biased angular velocity measurements has been treated in [7] assuming that the attitude is available for feedback. In [8], the attitude control problem has been addressed in the presence of unknown angular velocity bias, using IMU measurements, assuming that the rigid body inertia is known. In [9], [10], for instance, the attitude stabilization problem has been solved without attitude and angular velocity measurements and without the knowledge of the inertia matrix. The proposed control schemes rely directly on measurements in the body frame of some known inertial vectors. The extension to the case of trajectory tracking remains an open problem. In [11] the adaptive attitude tracking problem, with unknown inertia, has been addressed using the measurement in the body frame of a single (non-constant) inertial vector, assuming perfect angular velocity measurements. This observer-based controller is mainly suitable for non-stationary flights such as in satellite applications.

In [12], the attitude tracking problem using IMU measurements, with unknown angular velocity bias and unknown inertia has been addressed. Two control laws were presented; the first one considers only the case of biased angular velocity measurements, and the second one is an extension to the case of unknown inertia matrix. The second controller which considers unknown inertia and gyrobias simultaneously, relies on the use of the attitude observer of [6] which provides attitude estimates to be used in the tracking control law. The overall certainty-equivalence-type adaptive control scheme

A. Benallegue is with CNRS-AIST Joint Robotics Laboratory (JRL), UMI3218/RL, Tsukuba, Japan. Y. Chitour is with Université Paris-Sud, CentraleSupélec, CNRS, France. A. Tayebi is with the Department of Electrical Engineering, Lakehead University, Thunder Bay, Ontario, Canada. benalleg@lisv.uvsq.fr, Yacine.CHITOUR@lss.supelec.fr,

atayebi@lakeheadu.ca that has been proposed (without proof) seems to rely on a conjectured separation principle.

In the present work, we aim to solve the attitude tracking problem in the case where 1) the rigid body inertia is unknown, 2) the measured angular velocity is biased with an unknown constant bias, and 3) the attitude is not directly available for measurement. To handle the three above mentioned constraints simultaneously, we derive an adaptive control scheme that relies only on biased angular velocity measurements and body-frame measurements of some known inertial vectors. The control design relies on a transformation that allows to linearly parameterize some terms in the system's vector field with respect to the unknown inertia matrix [3], [9], [13], [14]. Our approach is different from the one proposed in [12], and does not rely on the certainty-equivalence principle with separate observer/controller design, but rather relies on a direct injection of the measurements in the control law, and the stability of the interconnection observer-controller is proven as a whole. Moreover, the number of adaptations and the order of the proposed tracking controller are lower than that of [12].

\section{BACKGROUND}

\section{A. Preliminaries}

The quaternion set $\mathbb{Q}$ is a four-dimensional vector space over the reals, which forms a group with the quaternion multiplication denoted by " $\odot$ ", which is distributive, associative but not commutative. The multiplication of two quaternions $P=\left(p_{0}, p\right)$ and $Q=\left(q_{0}, q\right)$ is defined as

$$
P \odot Q=\left(p_{0} q_{0}-p^{T} q, p_{0} q+q_{0} p+p \times q\right),
$$

and has the quaternion $(1,0)$ as the identity element. Note that, for a given quaternion $Q=\left(q_{0}, q\right)$, one has $Q \odot Q^{-1}=Q^{-1} \odot Q=(1, \mathbf{0})$, where $Q^{-1}=\frac{\left(q_{0},-q\right)}{\|Q\|^{2}}$.

Note that in the case where $Q=\left(q_{0}, q\right)$ is a unit-quaternion, the inverse is given by $Q^{-1}=\left(q_{0},-q\right)$.

The unit quaternion $Q=\left(q_{0}, q\right)$, composed of a scalar component $q_{0} \in \mathbb{R}$ and a vector component $q \in \mathbb{R}^{3}$, represents the orientation of the inertial frame $\mathcal{I}$ with respect to the body-attached frame $\mathcal{B}$, and are subject to the constraint $q_{0}^{2}+q^{T} q=1$. The rotation matrix, related to the unit-quaternion $Q$, that brings the inertial frame into the body-attached frame, can be obtained through the Rodrigues formula $R=\mathcal{R}(Q)$ with the mapping $\mathcal{R}: \mathbb{S}^{3} \rightarrow S O(3)$ is defined as

$$
\begin{aligned}
\mathcal{R}(Q) & =I_{3}+2 q_{0} S(q)+2 S^{2}(q) \\
& =\left(q_{0}^{2}-q^{T} q\right) I_{3}+2 q q^{T}+2 q_{0} S(q)
\end{aligned}
$$

where $I_{3}$ is the 3-by-3 identity matrix and $S(x)$ is the skewsymmetric matrix associated to the vector $x \in \mathbb{R}^{3}$ such that $S(x) V=x \wedge V$ for any vector $V \in \mathbb{R}^{3}$, where $\wedge$ denotes the vector cross product of $\mathbb{R}^{3}$. Note that $\mathcal{R}(Q)=\mathcal{R}(-Q)$ for every $Q \in \mathbb{Q}$ and $\mathcal{R}$ defines a two-sheet covering of $S O(3)$ by $\mathbb{Q}$, i.e., for every $R \in S O(3)$ there exist exactly two distinct quaternions satisfying $\mathcal{R}(Q)=R$. As a consequence every vector field $f$ defined on $\mathbb{Q}$ so that $f(-Q)=-f(Q)$ for every $Q \in \mathbb{Q}$ defines a vector field $\tilde{f}$ on $S O(3)$. 
Throughout this paper, we will denote by $(0, X)$ the quaternion associated to the three-dimensional vector $X$. A vector $x_{\mathcal{I}}$ expressed in the inertial frame $\mathcal{I}$ can be expressed in the body frame $\mathcal{B}$ by $x_{\mathcal{B}}=R^{T} x_{\mathcal{I}}$ or equivalently in terms of unit-quaternion as $\left(0, x_{\mathcal{B}}\right)=$ $Q^{-1} \odot\left(0, x_{\mathcal{I}}\right) \odot Q$, where $Q$ is the unit-quaternion associated to $R$ by (2).

Let us define the following mapping vect $: \mathbb{R}^{n \times n} \rightarrow \mathbb{R}^{n^{2}}$, such that for a given matrix $A \in \mathbb{R}^{n \times n}$, we associate the vector $\operatorname{vect}(A)=$ $\left[v_{1}, \ldots, v_{n}\right]^{T}$, where $v_{i}, i=1, \ldots, n$, are the row vectors of the matrix $A$.

\section{B. Equations of motion}

In this work, we consider a rigid body whose rotational dynamics are governed by

$$
\Sigma_{R}:\left\{\begin{array}{l}
\dot{Q}=\frac{1}{2} Q \odot(0, \omega) \\
I_{b} \dot{\omega}=\tau-S(\omega) I_{b} \omega
\end{array}\right.
$$

where $\omega$ is the angular velocity of the rigid body expressed in the body-attached frame $\mathcal{B}, \tau$ is the external torque applied to the system expressed in $\mathcal{B}$ and $I_{b} \in \mathbb{R}^{3 \times 3}$ is a symmetric positive definite constant inertia matrix (assumed to be unknown) of the rigid body with respect to $\mathcal{B}$ of the form

$$
I_{b}=\left[\begin{array}{ccc}
I_{11} & I_{12} & I_{13} \\
I_{12} & I_{22} & I_{23} \\
I_{13} & I_{23} & I_{33}
\end{array}\right]
$$

\section{MAin Results}

\section{A. Problem statement}

Let us define the desired attitude trajectory in terms of the rotation matrix $R_{d}(t)$ governed by the following dynamics,

$$
\dot{R}_{d}=R_{d} S\left(\omega_{d}\right),
$$

with $\omega_{d}(t)$ being the desired angular velocity vector.

An equivalent desired unit-quaternion $Q_{d}(t)$ is defined as $R_{d}(t)=$ $\mathcal{R}\left(Q_{d}(t)\right)$. Its dynamics are governed by

$$
\dot{Q}_{d}=\frac{1}{2} Q_{d} \odot\left(0, \omega_{d}\right) .
$$

The following assumptions are used throughout the paper:

Assumption A1. The rigid body is equipped with sensors that provide measurements (in the body-attached frame) of constant and known inertial vectors $r_{i} \in \mathbb{R}^{3}, i=1, \ldots, n \geq 2$. At least two vectors, among the $n$ inertial vectors, are noncollinear. The vector measurements in the body-attached frame are denoted by $b_{i} \in \mathbb{R}^{3}, i=1, \ldots, n$. The vectors $r_{i}$ and $b_{i}$ are related by $b_{i}=R^{T} r_{i}$.

Assumption A2. The attitude ( $Q$ or $R$ ) is unknown (i.e., unavailable for feedback).

Assumption A3. The measured angular velocity is assumed to be biased, so that the relation between the actual and measured velocities is given by

$$
\omega=\omega_{m}+\delta
$$

where $\delta$ is the unknown constant bias, $\omega$ and $\omega_{m}$ are the actual and the measured velocity vectors respectively.

Assumption A4. The inertia matrix $I_{b}$ is assumed to be unknown.

Assumption A5. The desired angular velocity vector $\omega_{d}$ and its first to sixth derivatives are bounded.

Our objective is to design a control input $\tau$ guaranteeing Almost Global Asymptotic Convergence (AGAC) of the body attitude and angular velocity to their desired values, under the above assumptions.
This means that there exists an equilibrium point $E q$ (in the appropriate state space) such that, for almost every initial condition (with respect to the Lebesgue measure in the state space), the corresponding trajectory of the closed loop system converges to Eq.

\section{B. Linearly parameterized model for the control}

Let us consider Assumptions A3 and A4 and define the following parameters

$$
\begin{aligned}
\theta_{1} & =\delta \in \mathbb{R}^{3} \\
\theta_{2} & =S(\delta) I_{b} \delta \in \mathbb{R}^{3}, \\
\theta_{3} & =\left(I_{11}, I_{22}, I_{33}, I_{23}, I_{13}, I_{12}\right)^{T} \in \mathbb{R}^{6}, \\
\theta_{4} & =\operatorname{vect}\left(S(\delta) I_{b}-S\left(I_{b} \delta\right)\right) \in \mathbb{R}^{9}, \\
\Theta^{T} & =\left[\begin{array}{lll}
\theta_{2}^{T} & \theta_{3}^{T} & \theta_{4}^{T}
\end{array}\right] \in \mathbb{R}^{18} .
\end{aligned}
$$

Using the second equation of (3), we can write the following $I_{b}\left(\dot{\omega}-\dot{\omega}_{d}\right)=-\left(S\left(\omega_{m}\right) F_{1}\left(\omega_{m}\right)+F_{1}\left(\dot{\omega}_{d}\right)\right) \theta_{3}-F_{2}\left(\omega_{m}\right) \theta_{4}-\theta_{2}+\tau$,

where $F_{1}(\omega)$ is defined as

$$
F_{1}(\omega)=\left[\begin{array}{cccccc}
\omega_{1} & 0 & 0 & 0 & \omega_{3} & \omega_{2} \\
0 & \omega_{2} & 0 & \omega_{3} & 0 & \omega_{1} \\
0 & 0 & \omega_{3} & \omega_{2} & \omega_{1} & 0
\end{array}\right]
$$

and $F_{2}(\omega)$ as

$$
F_{2}(\omega)=\left[\begin{array}{ccccccccc}
\omega_{1} & \omega_{2} & \omega_{3} & 0 & 0 & 0 & 0 & 0 & 0 \\
0 & 0 & 0 & \omega_{1} & \omega_{2} & \omega_{3} & 0 & 0 & 0 \\
0 & 0 & 0 & 0 & 0 & 0 & \omega_{1} & \omega_{2} & \omega_{3}
\end{array}\right]
$$

The model given by Equation (5) can be written in a linear parameterizations form as

$$
I_{b}\left(\dot{\omega}-\dot{\omega}_{d}\right)=-G\left(\omega_{m}, \dot{\omega}_{d}\right) \Theta+\tau,
$$

with

$G\left(\omega_{m}, \dot{\omega}_{d}\right)=\left[\begin{array}{ll}I_{3} & S\left(\omega_{m}\right) F_{1}\left(\omega_{m}\right)+F_{1}\left(\dot{\omega}_{d}\right) \quad F_{2}\left(\omega_{m}\right)\end{array}\right] \in \mathbb{R}^{3 \times 18}$

We also assume that $\omega_{d}$ verifies the following additional assumption.

Assumption A6. We assume that

$$
\limsup _{t \rightarrow \infty}\left|\operatorname{det} J_{\delta}\left(\omega_{d}, \dot{\omega}_{d}\right)\right|>0,
$$

where the $15 \times 15$ matrix-valued function $J_{\delta}\left(\omega_{d}, \dot{\omega}_{d}\right)$ is given by

$$
J_{\delta}\left(\omega_{d}, \dot{\omega}_{d}\right)=\left[\begin{array}{c}
\frac{d}{d t}\left[H_{\delta}\left(\omega_{d}, \dot{\omega}_{d}\right)\right] \\
\vdots \\
\frac{d^{5}}{d t^{5}}\left[H_{\delta}\left(\omega_{d}, \dot{\omega}_{d}\right)\right]
\end{array}\right] .
$$

where $H_{\delta}\left(\omega_{d}, \dot{\omega}_{d}\right)$ is a $3 \times 15$ matrix-valued function of the time $t$ given by

$$
H_{\delta}\left(\omega_{d}, \dot{\omega}_{d}\right)=\left[\begin{array}{ll}
S\left(\omega_{d}-\delta\right) F_{1}\left(\omega_{d}-\delta\right)+F_{1}\left(\dot{\omega}_{d}\right) & F_{2}\left(\omega_{d}-\delta\right)
\end{array}\right] .
$$

Assumption A6 is tailored to insure the following convergence result.

Lemma 1. Let $\delta \in \mathbb{R}^{3}$ and $\omega_{d}:[0, \infty) \rightarrow \mathbb{R}^{3}$ satisfying Assumption A6. Assume that there exists a measurable function $\Psi:[0, \infty) \rightarrow \mathbb{R}^{15}$ such that $\lim _{t \rightarrow \infty} J_{\delta}\left(\omega_{d}, \dot{\omega}_{d}\right) \Psi(t)=0$. Then $\liminf _{t \rightarrow \infty} \Psi(t)=0$.

Proof: Assumption A6 implies that $\liminf _{t \rightarrow \infty}\left\|J_{\delta}^{-1}\right\|$ is finite and since $\Psi=J_{\delta}^{-1}\left(J_{\delta} \Psi\right)$ one immediately deduces the conclusion.

Remark 1. Assumption A6 can be seen as a persistence of excitation condition for the biased desired angular velocity $\omega_{d}-\delta$ together with its first six time derivatives. 


\section{Control design}

Define $n$ vectors $b_{i}^{d}$ and $n$ vectors $\hat{b}_{i}$ corresponding to the desired and estimated vectors such that $b_{i}^{d}=R_{d}^{T} r_{i}$ and $\hat{b}_{i}=\hat{R}^{T} r_{i}$, for $i=1, \ldots, n$.

According to the model given by (6), we propose the following adaptive control law

$$
\tau=G\left(\omega_{m}, \dot{\omega}_{d}-\dot{\hat{\theta}}_{1}\right) \hat{\Theta}+z_{\gamma}-\alpha \bar{\omega},
$$

with $\bar{\omega}=\omega_{m}+\hat{\theta}_{1}-\omega_{d}$ and

$$
z_{\gamma}=\sum_{i=1}^{n} \gamma_{i} S\left(b_{i}^{d}\right) b_{i} ; z_{\rho}=\sum_{i=1}^{n} \rho_{i} S\left(\hat{b}_{i}\right) b_{i},
$$

where $\alpha>0, \gamma_{i}>0$ and $\rho_{i}>0$ are constant scalar gains.

The attitude estimator is given by

$$
\dot{\hat{Q}}=\frac{1}{2} \hat{Q} \odot(0, \hat{\omega})
$$

with $\hat{\omega}=\omega_{m}+\hat{\theta}_{1}-z_{\rho}$.

The adaptation scheme is given by

$$
\begin{aligned}
& \dot{\hat{\theta}}_{1}=\Gamma_{1} \operatorname{Proj}\left(-\left(z_{\gamma}+z_{\rho}\right), \hat{\theta}_{1}, \theta_{m}\right), \\
& \dot{\hat{\Theta}}=\Gamma_{2} \operatorname{Proj}\left(-G\left(\omega_{m}, \dot{\omega}_{d}-\dot{\hat{\theta}}_{1}\right)^{T} \bar{\omega}, \hat{\Theta}, \Theta_{m}\right),
\end{aligned}
$$

with $\left\|\hat{\theta}_{1}(0)\right\| \leq \theta_{m}$ and $\|\hat{\Theta}(0)\| \leq \Theta_{m}$. The matrices $\Gamma_{1}$ and $\Gamma_{2}$ are real symmetric positive definite. The positive parameters $\theta_{m}$ and $\Theta_{m}$ are the upper bounds of $\theta_{1}$ and $\Theta$, i.e., $\left\|\theta_{1}\right\| \leq \theta_{m},\|\Theta\| \leq \Theta_{m}$. The projection operator Proj is defined on $\mathbb{R}^{n} \times \mathbb{R}^{n} \times \mathbb{R}_{+}$as follows:

$$
\operatorname{Proj}\left(x, \hat{y}, y_{0}\right)=x-\frac{\eta_{1} \eta_{2}}{4\left(\epsilon^{2}+2 \epsilon y_{0}\right)^{n+1} y_{0}^{2}} \hat{y},
$$

with

$$
\begin{aligned}
& \eta_{1}=\left\{\begin{array}{l}
\left(\hat{y}^{T} \hat{y}-y_{0}^{2}\right)^{n+1} \\
0 \text { otherwise, }
\end{array} \text { if } \hat{y}^{T} \hat{y}>y_{0}^{2},\right. \\
& \eta_{2}=0.5 \hat{y}^{T} x+\sqrt{\left(0.5 \hat{y}^{T} x\right)^{2}+\mu^{2}}
\end{aligned}
$$

where $\epsilon$ and $\mu$ are arbitrary real positive constants and $n$ is an arbitrary positive integer. Let $\bar{y}$ be a constant vector in $B_{y_{0}}=\{y \in$ $\left.\mathbb{R}^{n} \mid\|y\| \leq y_{0}\right\}, \hat{y}(0) \in B_{y_{0}}$ and $\tilde{y}=\bar{y}-\hat{y}$. Consider the adaptation algorithm $\dot{\hat{y}}=\operatorname{Proj}\left(x, \hat{y}, y_{0}\right)$, then the following properties hold $\mid 15$ for every $t \geq 0$ :

$\left.P_{1}\right) \quad\|\hat{y}(t)\| \leq y_{0}+\epsilon$,

$\left.P_{2}\right)-\tilde{y}(t)^{T} \operatorname{Proj}\left(-x, \hat{y}(t), y_{0}\right) \leq x^{T} \tilde{y}(t)$.

$\left.P_{3}\right) \operatorname{Proj}\left(x, \hat{y}, y_{0}\right) \in \mathcal{C}^{n}$.

It is worth pointing out that the choice of this smooth projection algorithm is motivated by some technical reasons in the proof of our theorem that will be provided later. In fact, we will require the parameters estimates to be at least six times differentiable, and hence the integer $n$ involved in the projection mechanism has to satisfy $n \geq 6$.

\section{Convergence analysis}

Let us define the estimation error $\bar{R}=R \hat{R}^{T}$ and the tracking error $\tilde{R}=R R_{d}^{T}$ of the attitude that correspond to the unit quaternion errors $\bar{Q}=Q \odot \hat{Q}^{-1} \equiv\left(\bar{q}_{0}, \bar{q}\right)$ and $\tilde{Q}=Q \odot Q_{d}^{-1} \equiv\left(\tilde{q}_{0}, \tilde{q}\right)$ respectively. The estimation error dynamics are given by

$$
\dot{\bar{Q}}=\left[\begin{array}{c}
\dot{\bar{q}}_{0} \\
\dot{\bar{q}}
\end{array}\right]=\left[\begin{array}{c}
-\frac{1}{2} \bar{q}^{T} \hat{R}(\omega-\hat{\omega}) \\
\frac{1}{2}\left(\bar{q}_{0} I+S(\bar{q})\right) \hat{R}(\omega-\hat{\omega})
\end{array}\right],
$$

with $\omega-\hat{\omega}=z_{\rho}+\tilde{\theta}_{1}$.

The tracking error dynamics are given by

$$
\dot{\tilde{Q}}=\left[\begin{array}{c}
\dot{\tilde{q}}_{0} \\
\dot{\tilde{q}}
\end{array}\right]=\left[\begin{array}{c}
-\frac{1}{2} \tilde{q}^{T} R_{d}\left(\omega-\omega_{d}\right) \\
\frac{1}{2}\left(\tilde{q}_{0} I+S(\tilde{q})\right) R_{d}\left(\omega-\omega_{d}\right)
\end{array}\right],
$$

where $\omega-\omega_{d}=\bar{\omega}+\tilde{\theta}_{1}$, with $\tilde{\theta}_{1}=\theta_{1}-\hat{\theta}_{1}$.

Before stating our main results, we recall the following useful lemma given in [16] that will be used throughout the paper.

Lemma 2. Assume that there are $n$ vectors $b_{i}, i=1, \ldots, n$ measured in the body attached frame, corresponding to $n$ known inertial vectors $r_{i}, i=1, \ldots, n$. Assume that the constant parameters $\gamma_{i}$ and $\rho_{i}$ are strictly positive and at least two vectors among the $r_{i}$ vectors are non-collinear. Then, the following properties hold

1) The vectors $z_{\gamma}$ and $z_{\rho}$ satisfy

$$
\begin{gathered}
z_{\gamma} \equiv \sum_{i=1}^{n} \gamma_{i} S\left(b_{i}^{d}\right) b_{i}=-2 R_{d}^{T}\left(\tilde{q}_{0} I-S(\tilde{q})\right) W_{\gamma} \tilde{q}, \\
z_{\rho} \equiv \sum_{i=1}^{n} \rho_{i} S\left(\hat{b}_{i}\right) b_{i}=-2 \hat{R}^{T}\left(\bar{q}_{0} I-S(\bar{q})\right) W_{\rho} \bar{q},
\end{gathered}
$$

where the matrices $W_{\gamma}=-\sum_{i=1}^{n} \gamma_{i} S\left(r_{i}\right)^{2}$ and $W_{\rho}=$ $-\sum_{i=1}^{n} \rho_{i} S\left(r_{i}\right)^{2}$ are real symmetric and positive definite. If the gains $\gamma_{i}, \rho_{i}, i=1, \ldots, n$, are such that $W_{\gamma}$ and $W_{\rho}$ have two by two distinct eigenvalues, the following holds true.

2) $z_{\gamma}=0$ is equivalent to $\left(\tilde{q}_{0}=0, \tilde{q}=v_{\gamma}\right)$ or $\left(\tilde{q}_{0}= \pm 1, \tilde{q}=0\right)$, where $v_{\gamma}$ is a unit eigenvector of $W_{\gamma}$.

3) $z_{\rho}=0$ is equivalent to $\left(\bar{q}_{0}=0, \bar{q}=v_{\rho}\right)$ or $\left(\bar{q}_{0}= \pm 1, \bar{q}=0\right)$, where $v_{\rho}$ is a unit eigenvector of $W_{\rho}$.

The closed loop attitude error dynamics are given by

$$
\begin{gathered}
\dot{\tilde{Q}}=\left[\begin{array}{c}
\dot{\tilde{q}}_{0} \\
\dot{\tilde{q}}
\end{array}\right]=\left[\begin{array}{c}
-\frac{1}{2} \tilde{q}^{T} R_{d}\left(\bar{\omega}+\tilde{\theta}_{1}\right) \\
\frac{1}{2}\left(\tilde{q}_{0} I+S(\tilde{q})\right) R_{d}\left(\bar{\omega}+\tilde{\theta}_{1}\right)
\end{array}\right] \\
\dot{\bar{Q}}=\left[\begin{array}{c}
\dot{\bar{q}} 0 \\
\dot{\bar{q}}
\end{array}\right]=\left[\begin{array}{c}
\bar{q}_{0} \bar{q}^{T} W_{\rho} \bar{q}-\frac{1}{2} \bar{q}^{T} \hat{R} \tilde{\theta}_{1} \\
-\left(I-\bar{q} \bar{q}^{T}\right) W_{\rho} \bar{q}+\frac{1}{2}\left(\bar{q}_{0} I+S(\bar{q})\right) \hat{R} \tilde{\theta}_{1}
\end{array}\right] \\
I_{b} \dot{\bar{\omega}}=-\alpha \bar{\omega}+z_{\gamma}-G\left(\omega_{m}, \dot{\omega}_{d}-\dot{\hat{\theta}}_{1}\right) \tilde{\Theta} \\
\dot{\tilde{\theta}}_{1}=-\Gamma_{1} \operatorname{Proj}\left(-\left(z_{\gamma}+z_{\rho}\right), \hat{\theta}_{1}, \theta_{m}\right) \\
\dot{\tilde{\Theta}}=-\Gamma_{2} \operatorname{Proj}\left(-G\left(\omega_{m}, \dot{\omega}_{d}-\dot{\hat{\theta}}_{1}\right)^{T} \bar{\omega}, \hat{\Theta}, \Theta_{m}\right)
\end{gathered}
$$

where $\tilde{\theta}_{1}=\theta_{1}-\hat{\theta}_{1}$ and $\tilde{\Theta}=\Theta-\hat{\Theta}$.

Note that these dynamics are non-autonomous. Define $X=$ $\left(\tilde{Q}, \bar{Q}, \bar{\omega}, \tilde{\theta}_{1}, \tilde{\Theta}\right)$ in the state space $\mathcal{X}:=\mathbb{S}^{3} \times \mathbb{S}^{3} \times \mathbb{R}^{3} \times \mathbb{R}^{3} \times \mathbb{R}^{18}$. Note that $\mathcal{X}$ has dimension 30 . The above dynamics can be written as

$$
\dot{X}=f\left(X, R_{d}(t), \omega_{d}(t), \dot{\omega}_{d}(t)\right),
$$

where $f$ is a time-varying vector field defined on $\mathcal{X}$. Now, one can state our main result in the following theorem:

Theorem 1. Consider the rigid body dynamics (3) with the adaptive control scheme (8)-(10), resulting in the closed loop attitude error dynamics given by (16)-20. Then under Assumptions A1-A5 and the gains $\gamma_{i}, \rho_{i}, i=1, \ldots, n$, are chosen such that $W_{\gamma}$ and $W_{\rho}$ have two by two distinct eigenvalues, all the signals of the closed loop-system are bounded and

i) $\lim _{t \rightarrow \infty}\left(\tilde{\theta}_{1}(t), \omega(t)-\omega_{d}(t)\right)=(0,0), \lim _{t \rightarrow \infty}\left(\tilde{q}_{0}(t), \tilde{q}(t)\right)=$ $\left(( \pm 1,0)\right.$ or $\left.\left(0, v_{\gamma}\right)\right) \quad$ and $\quad \lim _{t \rightarrow \infty}\left(\bar{q}_{0}(t), \bar{q}(t)\right) \quad=$ $\left(( \pm 1,0)\right.$ or $\left.\left(0, v_{\rho}\right)\right)$ where $v_{\gamma}$ and $v_{\rho}$ are, respectively, the unit eigenvectors of $W_{\gamma}$ and $W_{\rho}$.

ii) The undesired equilibria characterized by $\bar{q}_{0}=0$ and/or $\tilde{q}_{0}=0$ are unstable.

iii) If, in addition Assumption A6 is satisfied, then $\lim _{t \rightarrow \infty} \tilde{\Theta}(t)=$ 0 .

iv) Moreover, if the gain matrices $W_{\gamma}$ and $W_{\rho}$ satisfy the following condition

$$
4 \lambda_{\min }(M)-\operatorname{Tr} M-\alpha \operatorname{Tr} I_{b}^{-1}>0, \text { for } M \in\left\{W_{\gamma}, W_{\rho}\right\},
$$


then, for almost any initial condition $X_{0} \in \mathcal{X}$, the corresponding trajectory of 21] converges to a point of $\widetilde{\Omega}_{1}=$ $\{(( \pm 1,0),( \pm 1,0), 0,0,0)\}$.

Proof. Let us consider the following Lyapunov function candidate

$$
\begin{aligned}
V & =2 \tilde{q}^{T} W_{\gamma} \tilde{q}+2 \bar{q}^{T} W_{\rho} \bar{q}+\frac{1}{2} \bar{\omega}^{T} I_{b} \bar{\omega} \\
& +\frac{1}{2} \tilde{\theta}_{1}^{T} \Gamma_{1}^{-1} \tilde{\theta}_{1}+\frac{1}{2} \tilde{\Theta}^{T} \Gamma_{2}^{-1} \tilde{\Theta}
\end{aligned}
$$

The time derivative of $(23)$, in view of $(16,, 17),(18)$ and Property $P_{2}$ ) of the projection operator, is given by

$$
\dot{V} \leq-\alpha \bar{\omega}^{T} \bar{\omega}-z_{\rho}^{T} z_{\rho} .
$$

According to 24, $V$ is non-increasing along trajectories of the dynamical system, implying that $\bar{\omega}, \bar{q}, \tilde{q}, \tilde{\theta}_{1}$ and $\tilde{\Theta}$ are bounded and $V$ converges to a non negative limit. One checks easily that $\ddot{V}$ is bounded for every trajectory of the system, implying that $\dot{V}$ is uniformly continuous, hence that $\dot{V} \rightarrow 0$. On the other hand, the time derivative of $z_{\rho}$ and $z_{\gamma}$ are given by

$$
\begin{aligned}
& \dot{z}_{\rho}=-S\left(\omega-\tilde{\theta}_{1}\right) z_{\rho}+\left(\sum_{i=1}^{n} \rho_{i} S\left(\hat{b}_{i}\right) S\left(b_{i}\right)\right)\left(z_{\rho}+\tilde{\theta}_{1}\right), \\
& \dot{z}_{\gamma}=-S\left(\omega_{d}\right) z_{\gamma}+\left(\sum_{i=1}^{n} \rho_{i} S\left(b_{i}^{d}\right) S\left(b_{i}\right)\right)\left(\omega-\omega_{d}\right),
\end{aligned}
$$

which are clearly bounded. Since, for any given trajectory of the closed loop system, $\ddot{V}$ is bounded, one deduces that $\bar{\omega} \rightarrow 0$ and $z_{\rho} \rightarrow 0$ as time tends to infinity. Using Lemma 2 , one sees that $\left(\bar{q}_{0} I-S(\bar{q})\right) W_{\rho} \bar{q} \rightarrow 0$ as time tends to infinity, which implies that either $\left(\bar{q}_{0}, \bar{q}\right) \rightarrow( \pm 1,0)$ or $\left(\bar{q}_{0}, \bar{q}\right) \rightarrow\left(0, v_{\rho}\right)$ where $v_{\rho}$ is a unit eigenvector of $W_{\rho}$. Since $\ddot{\bar{q}}_{0}$ and $\ddot{\bar{q}}$ are bounded, and in view of the previous results, it is clear that $\dot{\bar{q}}_{0} \rightarrow 0$ and $\dot{\bar{q}} \rightarrow 0$. Hence, in view of (17) one can conclude that $\tilde{\theta}_{1} \rightarrow 0$ then $t$ tends to infinity. Using (19), and the fact that $z_{\rho} \rightarrow 0$ and $\dot{z}_{\gamma}$ is bounded, it can be concluded that $\dot{\tilde{\theta}}_{1} \rightarrow 0$ and therefore $z_{\gamma} \rightarrow 0$. Using Lemma 2 one concludes that either $\left(\tilde{q}_{0}, \tilde{q}\right) \rightarrow( \pm 1,0)$ or $\left(\tilde{q}_{0}, \tilde{q}\right) \rightarrow\left(0, v_{\gamma}\right)$ as $t$ tends to infinity, where $v_{\gamma}$ is a unit eigenvector of $W_{\gamma}$. We just proved item (i) of the Theorem.

Now, let us prove item (ii). From earlier proven facts, it is clear that $\lim _{t \rightarrow \infty} \tilde{\Theta}(t)=\Theta_{l}$ with $\left\|\Theta_{l}\right\| \leq 2 \Theta_{m}+\epsilon$. The undesired equilibria characterized by $\bar{q}_{0}=0$ and/or $\tilde{q}_{0}=0$ are given by $X_{1}=$ $\left(v_{\gamma}, v_{\rho}, 0,0, \Theta_{l}\right), X_{2}=\left(v_{\gamma}, 0,0,0, \Theta_{l}\right)$ and $X_{3}=\left(0, v_{\rho}, 0,0, \Theta_{l}\right)$. Let us show that $\left.X_{1}=\left(\tilde{q}=v_{\gamma}, \bar{q}=v_{\rho}\right), \bar{\omega}=0, \tilde{\theta}_{1}=0, \tilde{\Theta}=\Theta_{l}\right)$ is unstable. The proof of instability of $X_{2}$ and $X_{3}$ follow similar steps and hence omitted here.

The proof consists in showing that there exists $X_{1}^{*}=$ $\left(\left(\tilde{q}_{0}^{*}, \tilde{q}^{*}\right),\left(\bar{q}_{0}^{*}, \bar{q}^{*}\right), \bar{\omega}^{*}, \tilde{\theta}_{1}^{*}, \tilde{\Theta}^{*}\right) \in \mathcal{X}$ arbitrarily close to $X_{1}$ such that $V\left(\tilde{q}^{*}, \bar{q}^{*}, \bar{\omega}^{*}, \tilde{\theta}_{1}^{*}, \tilde{\Theta}^{*}\right)<V\left(v_{\gamma}, v_{\rho}, 0,0, \Theta_{l}\right)$. This, with the fact that $V$ is non-increasing on $\mathcal{X}$ proves the instability of $X_{1}$.

Let us apply small rotations on the unit-quaternion $\left(0, v_{\gamma}\right)$ and $\left(0, v_{\rho}\right)$; that is

$$
\begin{aligned}
& \left(\begin{array}{c}
\tilde{q}_{0}^{*} \\
\tilde{q}^{*}
\end{array}\right)=\left(\begin{array}{c}
0 \\
v_{\gamma}
\end{array}\right) \odot\left(\begin{array}{c}
\tilde{\eta}_{0} \\
\tilde{\eta}
\end{array}\right)=\left(\begin{array}{c}
-\tilde{\eta}^{T} v_{\gamma} \\
\tilde{\eta}_{0} v_{\gamma}+S\left(v_{\gamma}\right) \tilde{\eta}
\end{array}\right) . \\
& \left(\begin{array}{c}
\bar{q}_{0}^{*} \\
\bar{q}^{*}
\end{array}\right)=\left(\begin{array}{c}
0 \\
v_{\rho}
\end{array}\right) \odot\left(\begin{array}{c}
\bar{\eta}_{0} \\
\bar{\eta}
\end{array}\right)=\left(\begin{array}{c}
-\bar{\eta}^{T} v_{\rho} \\
\bar{\eta}_{0} v_{\rho}+S\left(v_{\rho}\right) \bar{\eta}
\end{array}\right) .
\end{aligned}
$$

Letting $\Delta V=V\left(\tilde{q}^{*}, \bar{q}^{*}, \bar{\omega}^{*}, \tilde{\theta}_{1}^{*}, \tilde{\Theta}^{*}\right)-V\left(v_{\gamma}, v_{\rho}, 0,0, \Theta_{l}\right)$, one gets

$$
\begin{aligned}
\Delta V & =+\frac{1}{2} \bar{\omega}^{* T} I_{b} \bar{\omega}^{*}+\frac{1}{2} \tilde{\theta}_{1}^{* T} \Gamma_{1}^{-1} \tilde{\theta}_{1}^{*}+\frac{1}{2} \tilde{\Theta}^{* T} \Gamma_{2}^{-1} \tilde{\Theta}^{*} \\
& -\frac{1}{2} \tilde{\Theta}_{l}^{T} \Gamma_{2}^{-1} \tilde{\Theta}_{l}+2\left(\tilde{\eta}_{0}^{2}-1\right) \lambda_{\gamma}+2\left(S(\tilde{\eta}) v_{\gamma}\right)^{T} W_{\gamma}\left(S(\tilde{\eta}) v_{\gamma}\right) \\
& +2\left(\bar{\eta}_{0}^{2}-1\right) \lambda_{\rho}+2\left(S(\bar{\eta}) v_{\rho}\right)^{T} W_{\rho}\left(S(\bar{\eta}) v_{\rho}\right)
\end{aligned}
$$

where we used the fact that $W_{\gamma} v_{\gamma}=\lambda_{\gamma} v_{\gamma}$ and $W_{\rho} v_{\rho}=\lambda_{\rho} v_{\rho}$ since $v_{\gamma}$ and $v_{\rho}$ are the unit eigenvectors associated, respectively, to $\lambda_{\gamma}$ and $\lambda_{\rho}$.

Let us pick $\tilde{\eta}=\tilde{\epsilon} v_{\gamma}$ and $\bar{\eta}=\bar{\epsilon} v_{\rho}$, with $|\tilde{\epsilon}|$ and $|\bar{\epsilon}|$ arbitrarily small. The unit-quaternion $\left(\tilde{\eta}_{0}, \tilde{\eta}\right)=\left(1-\tilde{\epsilon}^{2}, \tilde{\epsilon} v_{\gamma}\right)$, corresponds to a rotation about $v_{\gamma}$ by an angle $\theta_{\tilde{\epsilon}}=2 \arcsin \tilde{\epsilon}$. Similarly, The unit-quaternion $\left(\bar{\eta}_{0}, \bar{\eta}\right)=\left(1-\bar{\epsilon}^{2}, \bar{\epsilon} v_{\rho}\right)$, corresponds to a rotation about $v_{\rho}$ by an angle $\theta_{\bar{\epsilon}}=2 \arcsin \bar{\epsilon}$.

With this choice, 27) leads to

$$
\begin{aligned}
\Delta V & =\quad+\frac{1}{2} \bar{\omega}^{* T} I_{b} \bar{\omega}^{*}+\frac{1}{2} \tilde{\theta}_{1}^{* T} \Gamma_{1}^{-1} \tilde{\theta}_{1}^{*}+\frac{1}{2} \tilde{\Theta}^{* T} \Gamma_{2}^{-1} \tilde{\Theta}^{*} \\
& -\quad \frac{1}{2} \tilde{\Theta}_{l}^{T} \Gamma_{2}^{-1} \tilde{\Theta}_{l}+2\left(\tilde{\eta}_{0}^{2}-1\right) \lambda_{\gamma}+2\left(\bar{\eta}_{0}^{2}-1\right) \lambda_{\rho} .
\end{aligned}
$$

It follows that $\Delta V<0$ as long as

$$
\begin{aligned}
& \tilde{\epsilon}^{2}=1-\tilde{\eta}_{0}^{2}> \\
& \frac{1}{4 \lambda_{\gamma}}\left(\bar{\omega}^{* T} I_{b} \bar{\omega}^{*}+\tilde{\theta}_{1}^{* T} \Gamma_{1}^{-1} \tilde{\theta}_{1}^{*}+\tilde{\Theta}^{* T} \Gamma_{2}^{-1} \tilde{\Theta}^{*}-\tilde{\Theta}_{l}^{T} \Gamma_{2}^{-1} \tilde{\Theta}_{l}\right),
\end{aligned}
$$

or

$$
\begin{aligned}
& \bar{\epsilon}^{2}=1-\bar{\eta}_{0}^{2}> \\
& \frac{1}{4 \lambda_{\rho}}\left(\bar{\omega}^{* T} I_{b} \bar{\omega}^{*}+\tilde{\theta}_{1}^{* T} \Gamma_{1}^{-1} \tilde{\theta}_{1}^{*}+\tilde{\Theta}^{* T} \Gamma_{2}^{-1} \tilde{\Theta}^{*}-\tilde{\Theta}_{l}^{T} \Gamma_{2}^{-1} \tilde{\Theta}_{l}\right),
\end{aligned}
$$

Consequently, there exist $\bar{\omega}^{*}, \tilde{\theta}_{1}^{*}, \tilde{\epsilon}$ and $\bar{\epsilon}$ arbitrarily small in magnitude, and $\tilde{\Theta}^{*}$ arbitrarily close to $\Theta_{l}$, such that $X_{1}^{*}$ is arbitrarily close to $X_{1}$ and $\Delta V<0$. Since the Lyapunov function $V$ is shown to be non-increasing, it is clear that $X_{1}$ is unstable.

Now, under the additional assumption A6, we prove item (iii). Notice that $\tilde{\Theta}^{T} \Gamma_{2}^{-1} \tilde{\Theta}$ admits a limit as $t$ tends to infinity and therefore, to prove the lemma it is enough to prove that $\liminf _{t \rightarrow \infty}\|\tilde{\Theta}(t)\|=0$. Notice also that the projection algorithm is smooth and all the parametric estimation errors are bounded as well as their first to sixth time derivatives. Since $\bar{\omega} \rightarrow 0$ and $\ddot{\bar{\omega}}$ is bounded, it is clear that $\dot{\bar{\omega}} \rightarrow 0$, which in view of 18 and the fact that $z_{\gamma} \rightarrow 0$ implies that $G\left(\omega_{m}, \dot{\omega}_{d}-\dot{\hat{\theta}}_{1}\right) \tilde{\Theta}$ tends to zero at $t$ goes to infinity. Defining

$$
G_{d}:=G\left(\omega_{d}-\theta_{1}, \dot{\omega}_{d}\right)=\left[\begin{array}{ll}
I_{3} & H_{\delta}\left(\omega_{d}, \dot{\omega}_{d}\right)
\end{array}\right],
$$

one can easily show that

$$
G\left(\omega_{m}, \dot{\omega}_{d}-\dot{\hat{\theta}}_{1}\right)-G_{d} \rightarrow 0
$$

as $t$ tends to infinity, since $\left(\omega-\omega_{d}\right) \rightarrow 0$ and $\dot{\hat{\theta}}_{1} \rightarrow 0$. Consequently, $G_{d} \tilde{\Theta} \rightarrow 0$ as $t$ goes to infinity. Since $\frac{d^{2}}{d t^{2}}\left(G_{d} \tilde{\Theta}\right)$ is bounded, one deduces that $\frac{d}{d t}\left(G_{d} \tilde{\Theta}\right)$ is uniformly continuous, and hence tends to zero as $t$ tends to infinity. Using Property P3), and the boundedness of all signals involved in the closed-loop system, one can easily show that $\dot{\tilde{\Theta}}$ tends to zero. The latter fact combined with the convergence to zero of $\frac{d}{d t}\left(G_{d} \tilde{\Theta}\right)$ yield that $\frac{d G_{d}}{d t} \tilde{\Theta}$ tends to zero as $t$ tends to infinity. One can also show that $G_{d} \tilde{\Theta}$ is sufficiently differentiable, thanks to property P3) of the projection algorithm. Therefore, by an easy induction argument, it can be concluded that, for $0 \leq k \leq 5$,

$$
\lim _{t \rightarrow \infty} \frac{d^{(k)} G_{d}}{d t^{k}} \tilde{\Theta}=0 .
$$

Setting $\Psi(t):=\left(\tilde{\theta}_{3}^{T}, \tilde{\theta}_{4}^{T}\right)^{T}$, one has

$$
G_{d} \tilde{\Theta}=\tilde{\theta}_{2}+H_{\delta}\left(\omega_{d}, \dot{\omega}_{d}\right) \Psi(t) .
$$

Differentiating (32) and using the fact that $\dot{\tilde{\theta}}_{2} \rightarrow 0$ and $\dot{\Psi} \rightarrow 0$, one concludes that $\frac{d}{d t} H_{\delta}\left(\omega_{d}, \dot{\omega}_{d}\right) \Psi \rightarrow 0$ as $t$ goes to infinity. Using similar arguments, one can show that $\frac{d^{k}}{d t^{k}} H_{\delta}\left(\omega_{d}, \dot{\omega}_{d}\right) \Psi \rightarrow 0$ as $t$ goes to infinity, for $1 \leq k \leq 5$. Consequently, $\lim _{t \rightarrow \infty} J_{\delta}\left(\omega_{d}, \dot{\omega}_{d}\right) \Psi(t)=$ 0 , where $J_{\delta}\left(\omega_{d}, \dot{\omega}_{d}\right)$ has been defined in Assumption A6. According to Lemma 11 it follows that $\lim _{t \rightarrow \infty} \Psi(t)=0$, and consequently, in view of $32, \lim _{t \rightarrow \infty} \tilde{\theta}_{2}(t)=0$. This proves that $\liminf _{t \rightarrow \infty}\|\tilde{\Theta}(t)\|=0$.

Right now, We have proved that 
- The trajectories of 21 converge to the following subsets of $\mathbb{S}^{3} \times \mathbb{S}^{3} \times \mathbb{R}^{3} \times \mathbb{R}^{3} \times \mathbb{R}^{18}$ given by

$$
\begin{aligned}
& \widetilde{\Omega}_{1}=\{(( \pm 1,0),( \pm 1,0), 0,0,0)\}, \\
& \widetilde{\Omega}_{2}=\left\{\left(( \pm 1,0),\left(0, v_{j \rho}\right), 0,0,0\right), j=1,2,3\right\}, \\
& \widetilde{\Omega}_{3}=\left\{\left(\left(0, v_{i \gamma}\right),( \pm 1,0), 0,0,0\right), i=1,2,3\right\}, \\
& \widetilde{\Omega}_{4}=\left\{\left(\left(0, v_{i \gamma}\right),\left(0, v_{j \rho}\right), 0,0,0\right), i=1,2,3 ; j=1,2,3\right\},
\end{aligned}
$$

with $v_{i \gamma}$ and $v_{j \rho}$ are unit eigenvectors of $W_{\gamma}$ and $W_{\rho}$ respectively for $1 \leq i \leq j \leq 3$.

- The equilibria of the subsets $\widetilde{\Omega}_{2}, \widetilde{\Omega}_{3}$ and $\widetilde{\Omega}_{4}$ are unstable.

Now, we will show that from almost all initial conditions, the closed loop trajectories will converge to $\widetilde{\Omega}_{1}$ if condition $\sqrt{22}$ is satisfied, which proves the last statement of the theorem.

Let $X_{e q}$ be an element in some $\widetilde{\Omega}_{i}$ and write a trajectory as

$$
x(\cdot)=X_{e q}+Z(\cdot)
$$

where

$$
Z=\left(Z_{\tilde{q}_{0}}, Z_{\tilde{q}}, Z_{\bar{q}_{0}}, Z_{\bar{q}}, Z_{\bar{\omega}}, Z_{\tilde{\theta}_{1}}, Z_{\tilde{\Theta}}\right)^{T} .
$$

First note that $\bar{\omega}=Z_{\bar{\omega}}$ and we set $z_{\gamma}=Z_{\gamma}$ and $z_{\rho}=Z_{\rho}$ with

$Z_{\gamma}=-2 R_{d}^{T}\left[\lambda_{\gamma} Z_{\tilde{q}_{0}} v_{\gamma}-S\left(v_{\gamma}\right) W_{\gamma} Z_{\tilde{q}}+Z_{\tilde{q}_{0}} W_{\gamma} Z_{\tilde{q}}-S\left(Z_{\tilde{q}}\right) W_{\gamma} Z_{\tilde{q}}\right]$ if $\tilde{Q}=\left(0, v_{\gamma}\right)$, and

$$
Z_{\gamma}=-2 R_{d}^{T}\left[W_{\gamma} Z_{\tilde{q}}+\left(Z_{\tilde{q}_{0}} I_{3}-S\left(Z_{\tilde{q}}\right)\right) W_{\gamma} Z_{\tilde{q}}\right],
$$

if $\tilde{Q}=(1,0)$ and

$$
\begin{aligned}
Z_{\rho}= & -2 \hat{R}^{T}\left[\lambda_{\rho}\left(Z_{\bar{q}_{0}} I_{3}-S\left(Z_{\bar{q}}\right)\right) v_{\rho}-S\left(v_{\rho}\right) W_{\rho} Z_{\bar{q}}\right. \\
& \left.+\left(Z_{\bar{q}_{0}} I_{3}-S\left(Z_{\bar{q}}\right)\right) W_{\rho} Z_{\bar{q}}\right]
\end{aligned}
$$

if $\bar{Q}=\left(0, v_{\rho}\right)$, and

$$
Z_{\rho}=-2 \hat{R}^{T}\left(W_{\rho} Z_{\bar{q}}+\left(Z_{\bar{q}_{0}} I_{3}-S\left(Z_{\bar{q}}\right)\right) W_{\rho} Z_{\bar{q}}\right),
$$

if $\bar{Q}=(1,0)$.

If $\tilde{Q}_{e q}=\left(0, v_{\gamma}\right)$, the corresponding quaternion constraint yields

$$
Z_{\tilde{q}_{0}}^{2}+\left\|v_{\gamma}+Z_{\tilde{q}}\right\|^{2}=1,
$$

and then

$$
\left(Z_{\tilde{q} 0}^{2}+\left\|Z_{\tilde{q}}\right\|^{2}\right)+2 v_{\gamma}^{T} Z_{\tilde{q}}=0 .
$$

Similarly, if $\bar{Q}_{e q}=\left(0, v_{\rho}\right)$, one deduces from the corresponding quaternion constraint that

$$
\left(Z_{\bar{q}_{0}}^{2}+\left\|Z_{\bar{q}}\right\|^{2}\right)+2 v_{\rho}^{T} Z_{\bar{q}}=0 .
$$

If $\tilde{Q}_{e q}=(1,0)$, the corresponding quaternion constraint yields

$$
\left(Z_{\tilde{q}_{0}}^{2}+\left\|Z_{\tilde{q}}\right\|^{2}\right)+2 Z_{\tilde{q}_{0}}=0,
$$

and similarly, if $\bar{Q}_{e q}=(1,0)$, one deduces from the corresponding quaternion constraint that

$$
\left(Z_{\bar{q}_{0}}^{2}+\left\|Z_{\bar{q}}\right\|^{2}\right)+2 Z_{\bar{q}_{0}}=0 .
$$

We will actually prove that the points of the state space converging to the undesired equilibrium points in $\widetilde{\Omega}_{2}, \widetilde{\Omega}_{3}$ and $\widetilde{\Omega}_{4}$, form a set of measure zero.

Consider, for instance, a point in $\tilde{\Omega}_{2}$, let say $X_{e q}=$ $\left((1,0),\left(0, v_{\rho}\right), 0,0,0\right)$ where $v_{\rho}$ is a unit-length eigenvector of $W_{\rho}$ If $x \in \mathbb{R}^{3}$, we use $x^{\perp}$ to denote the vector in the two-dimensional plane $v_{\rho}^{\perp}$ given by $x^{\perp}=x-\left(v_{\rho}^{T} x\right) v_{\rho}$ and $W_{\rho}^{\perp}$ the restriction of $W_{\rho}$ to $v_{\rho}^{\perp}$. Recall first that the dimension of the state space $\mathcal{X}$ is equal to 30 and, by using the equations 35 and 36 , one deduces that

$$
Z_{\tilde{q}_{0}}=-\frac{\left\|Z_{\tilde{q}}\right\|^{2}}{1+\sqrt{1-\left\|Z_{\tilde{q}}\right\|^{2}}}, \quad v_{\rho}^{T} Z_{\bar{q}}=-\frac{Z_{\bar{q}_{0}}^{2}+\left\|Z_{\bar{q}}^{\perp}\right\|^{2}}{1+\sqrt{1-\left(Z_{\bar{q}_{0}}^{2}+\left\|Z_{\bar{q}}^{\perp}\right\|^{2}\right)}} .
$$

The reduced variable $Z_{r e d}$ is given by

$$
Z_{\text {red }}=\left(Z_{\tilde{q}}, Z_{\bar{q}_{0}}, Z_{\bar{q}}^{\perp}, Z_{\bar{\omega}}, Z_{\tilde{\theta}_{1}}, Z_{\tilde{\Theta}}\right)^{T},
$$

belongs to a smooth manifold $\mathcal{M}_{e q}$ of dimension 30. Fix a neighborhood $\mathcal{N}$ of the origin for the reduced variable so that the projection operators are equal to the corresponding identity operators in $\mathcal{N}$. Then, as long as the corresponding trajectory lies in $\mathcal{N}$ it obeys to the following dynamics

$$
\dot{Z}_{\text {red }}=A(t) Z_{\text {red }}+F\left(t, Z_{\text {red }}\right),
$$

where $A(t)$ and $F\left(t, Z_{\text {red }}\right)$ are given in the appendix.

We decomposed the error dynamics in (38) into a linear part and a super linear one, i.e, there exists a positive constant $C_{0}$ such that $F$ verifies an estimate of the type

$$
\left\|F\left(t, Z_{\text {red }}\right)\right\| \leq C_{0}\left\|Z_{\text {red }}\right\|^{2}, \quad\left|\operatorname{div} F\left(t, Z_{\text {red }}\right)\right| \leq C_{0}\left\|Z_{\text {red }}\right\|,
$$

for every $\left(t, Z_{\text {red }}\right) \in \mathbb{R}_{+} \times \mathcal{M}_{e q}$. Note also that the time-varying matrix $A(\cdot)$ does not depend on $Z_{\text {red }}$ and its trace is constant and equal to

$$
\operatorname{Tr} A(t) \equiv 4 \lambda_{\rho}-\operatorname{Tr} W_{\rho}-\alpha \operatorname{Tr} I_{b}^{-1}:=\xi .
$$

Since the matrix $W_{\rho}$ satisfies 22], the right-hand side $\xi$ of (40) is strictly positive. Assume now that the conclusion of the theorem does not hold true and more particularly, that there exist a measurable subset set $J$ of $\mathcal{X}$ with positive measure such that all trajectories of 21] starting in $J$ converge to $X_{e q}$. Let $J(t)$, the image of $J$ at time $t$ by the flow $\psi(t, 0)$ of the reduced dynamics. Since $J(t)$ converges to $\left\{X_{e q}\right\}$ as $t$ tends to infinity, one can assume, with no loss of generality, that $J$ is chosen close enough to $X_{e q}$ so that $J(t)$ lies in the neighborhood $\mathcal{N}$ for every $t \geq 0$ and therefore $\psi(t, 0)$ is the flow associated with the time-varying equation (38). Moreover, if $m(J(t))$ denotes the measure of $J(t)$, then $m(J(t))$ must tend to zero as $t$ tends to infinity.

On the other hand, one has for $t \geq 0$,

$$
m(J(t))=\int_{J(t)} d Z_{t}=\int_{J}|\operatorname{det}(t, Z)| d Z,
$$

where $\operatorname{det}(t, Z)$ denotes the determinant of $D \psi(t, 0, Z)$, the differential of $\psi(t, 0, Z)$ with respect to the initial condition $Z \in J$. Recall that, for every $t \geq 0$, one has

$$
\frac{\partial d(t, Z)}{\partial t}=(\operatorname{Tr} A(t)+\operatorname{div} F(t, \psi(t, 0, Z))) d(t, Z), \quad d(0, Z)=1 .
$$

By taking into account 39 and 40 , one deduces that $\operatorname{det}(t, Z) \geq e^{\xi t / 2}$ for $t$ large enough, hence $m(J(t)) \geq e^{\xi t / 2} m(J)$ which tends to infinity as $t$ tends to infinity. We reached a contradiction.

For the other equilibrium points of $\widetilde{\Omega}_{2}, \widetilde{\Omega}_{3}$ and $\widetilde{\Omega}_{4}$, one proceeds similarly to show that there does not exist a measurable subset $J$ of $\mathcal{X}$ with positive measure and such that trajectories of 21] starting in $J$ would converge to that equilibrium point. Therefore, for almost any initial condition $X_{0} \in \mathcal{X}$, the corresponding trajectory of 21 converges to a point of $\tilde{\Omega}_{1}$.

The following proposition provides a useful result that will help in the design of the parameters $\gamma_{i}$ and $\rho_{i}$ satisfying 22,

Proposition 1. Let $r_{1}$ and $r_{2}$ be two non-collinear 3-dimensional vectors. Let $r_{3}=r_{1} \times r_{2}$ and $\theta \in(0, \pi)$ the angle between $r_{1}$ and $r_{2}$. Set $R_{i}=\left\|r_{i}\right\|>0$ for $i=1,2$ and $R_{3}=R_{1} R_{2} \sin (\theta)$. If $W_{\gamma}=-\sum_{i=1}^{3} \gamma_{i} S\left(r_{i}\right)^{2}$ defined in Lemma 2 , then the following hold true:

a) The eigenvalues of $W_{\gamma}$ are equal to $\lambda_{1}=R_{1}^{2} \gamma_{1}+R_{2}^{2} \gamma_{2}, \lambda_{2}=$ $R_{3}^{2} \gamma_{3}+\frac{\lambda_{1}}{2}(1+\sqrt{1-D})$ and $\lambda_{3}=R_{3}^{2} \gamma_{3}+\frac{\lambda_{1}}{2}(1-\sqrt{1-D})$, with $D=\frac{4 R_{1}^{2} R_{2}^{2} \gamma_{1} \gamma_{2} \sin ^{2}(\theta)}{\lambda_{1}^{2}}$.

b) Set $\eta=\frac{R_{2}^{2} \gamma_{2}}{R_{1}^{2} \gamma_{1}}$. Note that $D=\frac{4 \eta \sin ^{2}(\theta)}{(1+\eta)^{2}}$. Then, Condition 22 is satisfied, if the parameters $\gamma_{i}$ and $\alpha$ are chosen such that

$$
\eta \neq 1,4 R_{3}^{2} \gamma_{3}=\lambda_{1}(1+3 \sqrt{1-D}), \lambda_{1}>\frac{2 \alpha \operatorname{Tr} I_{b}^{-1}}{1-\sqrt{1-D}} .
$$


Proof. First of all, notice that $\left\|r_{3}\right\|=R_{3}>0$ and $0<D<1$ since $r_{1}$ and $r_{2}$ are non-collinear and $\eta \neq 1$. Set $e_{1}=\left[\begin{array}{lll}1 & 0 & 0\end{array}\right]^{T}, e_{2}=$ $[\cos (\theta) \sin (\theta) 0]^{T}, e_{3}=\left[\begin{array}{lll}0 & 0 & 1\end{array}\right]^{T}$ and $E=\left[\begin{array}{lll}e_{1} & e_{2} & e_{3}\end{array}\right]$ and $R_{r}=$ $\left[\begin{array}{lll}\frac{r_{1}}{R_{1}} & \frac{r_{2}}{R_{2}} & \frac{r_{3}}{R_{3}}\end{array}\right]$. Then the matrix $U=E R_{r}^{-1}$ is clearly orthogonal. Finally, set $\mu_{i}=R_{i}^{2} \gamma_{i}$ for $i=1,2,3$ and $W=-\sum_{i=1}^{3} \mu_{i} S\left(e_{i}\right)^{2}$. It is immediate to see that $W=U W_{\gamma} U^{T}$, implying that $W$ and $W_{\gamma}$ have the same eigenvalues. Moreover, a direct computation shows that

$$
\begin{aligned}
& W=\left(\sum_{i=1}^{3} \mu_{i}\right) I-\sum_{i=1}^{3} \mu_{i} e_{i} e_{i}^{T}= \\
& {\left[\begin{array}{ccl}
\mu_{3}+\mu_{2} \sin ^{2}(\theta) & -\mu_{2} \cos (\theta) \sin (\theta) & 0 \\
-\mu_{2} \cos (\theta) \sin (\theta) & \mu_{1}+\mu_{3}+\mu_{2} \cos ^{2}(\theta) & 0 \\
0 & 0 & \mu_{1}+\mu_{2}
\end{array}\right] .}
\end{aligned}
$$

Straightforward calculations lead to the result of item (a). We next define $\gamma_{2}$ and $\gamma_{3}$ according to the definition of $\eta$ and the second part of Eq.41. This choice implies that $\lambda_{3}<\lambda_{2}<\lambda_{1}$. Finally, the third part of Eq. 41. imposes a choice on $\gamma_{1}$ and yields that Condition 22. is satisfied.

\section{Simulation RESUlTs}

In this section, we present simulation results showing the effectiveness of the proposed adaptive attitude trajectory tracking controller. We have considered for the simulations the inertia matrix $I_{b}=$ $\operatorname{diag}(0.0797,0.0797,0.1490)$, the gyro bias $\delta=[0.2,0.1,-0.1]^{T}$ and the inertial vectors $r_{1}=[0,0,1]^{T}$ and $r_{2}=[1,1,1]^{T} / \sqrt{3}$. The simulation sampling time is $0.01 \mathrm{sec}$. The control parameters have been chosen as $\gamma_{1}=\gamma_{2}=2, \rho_{1}=\rho_{2}=10$ and $\alpha=2$. The adaptation gains are $\Gamma_{1}=1$ and $\Gamma_{2}=1$. The parameters of the projections are given by $n=6, \epsilon=0.1, \mu=0.1, \theta_{m}=0.3$ and $\Theta_{m}=0.3$. The desired angular velocity vector $\omega_{d}$ has been chosen to satisfy the persistency excitation condition mentioned in assumption A6. We performed two simulation tests Test1 and Test2 to show the performance of the proposed control scheme and confirm the avoidance of the unwinding phenomenon. In the first simulation test, we considered the following initial conditions: $\omega(0)=[0,0,0]^{T}$, $Q_{d}(0)=[0.8,0,0.6,0]^{T}, \hat{Q}(0)=[-0.8,0,0.6,0]^{T}$ and $Q(0)=$ $[1,0,0,0]^{T}$. In the second simulation test, we considered the same initial conditions except for $Q$, where we started the scalar part of the unit quaternion from a negative value, i.e., $Q(0)=[-1,0,0,0]^{T}$. Figure 1 shows the evolution of the four components of the unitquaternion tracking errors with respect to time for Test 1 and Test2, respectively. Figure 2 shows the evolution of the unit-quaternion estimation error with respect to time for Test 1 and Test 2 respectively. We can clearly see that the unwinding phenomenon is avoided since both equilibria given by $\tilde{\Omega}_{1}$ are asymptotically stable. Figures 3 and 4 show the norm of the input signals and the angular velocity tracking error respectively. Fig. 5 shows that the parameter error $\tilde{\theta}_{1}$ converges to zero relatively fast, while the rest of the parameter errors $\tilde{\Theta}$ converge to zero relatively slow. This is due to the fact that only $\tilde{\Theta}$ depends on the richness of the reference signal.

\section{Conclusion}

A new adaptive attitude tracking control scheme, relying on inertial vector measurements, has been proposed for rigid body systems with unknown inertia and unknown angular velocity bias. Global boundedness of the system state variables and almost global asymptotic convergence of the body attitude and angular velocity to their desired values are proven. The convergence of the adaptive parameters to their true values is guaranteed under some kind of persistency of excitation condition on the reference trajectories. Compared to [12], the proposed control scheme involves fewer parameter adaptations
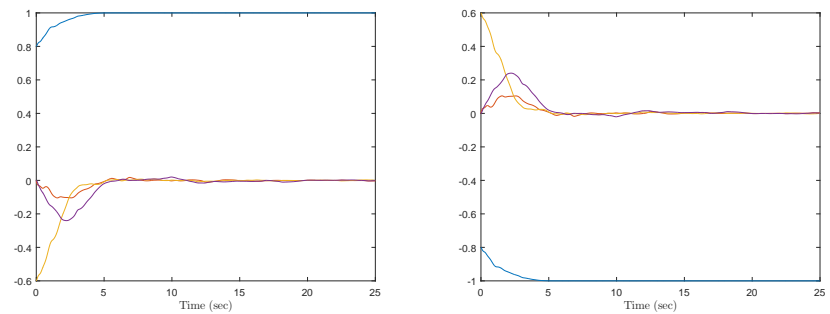

Fig. 1: Unit-quaternion tracking error $\tilde{Q}$ for Test 1 and Test2
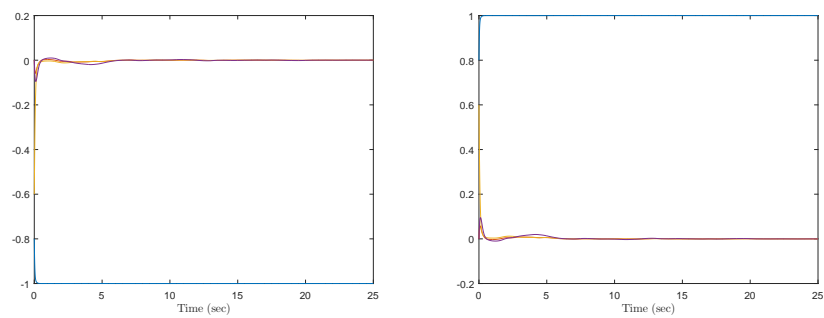

Fig. 2: Unit-quaternion estimation error $\bar{Q}$ for Test1 and Test2

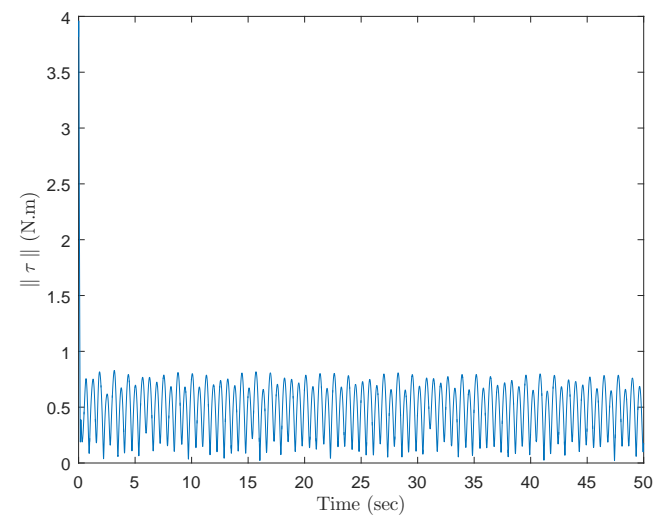

Fig. 3: Input torques $\|\tau\|$.

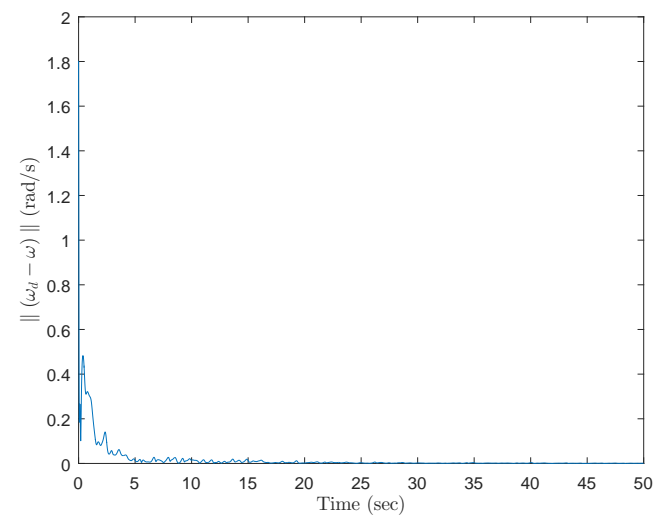

Fig. 4: Angular velocity errors $\left\|\omega-\omega_{d}\right\|$.

lower order dynamics and avoids the unwinding phenomenon. The performance of the proposed controller is illustrated through some simulation results. 


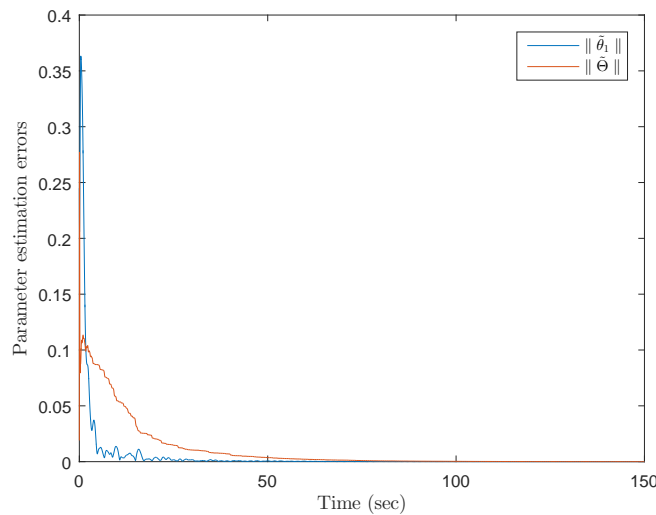

Fig. 5: Parameter estimation errors $\left\|\tilde{\theta}_{1}\right\|$ in blue and $\|\tilde{\Theta}\|$ in red.

\section{REFERENCES}

[1] B. Wie, H. Weiss, and A. Arapostathis, "Quarternion feedback regulator for spacecraft eigenaxis rotations," Journal of Guidance, Control, and Dynamics, vol. 12, no. 3, pp. 375-380, 2017/02/14 1989. [Online]. Available: http://dx.doi.org/10.2514/3.20418

[2] N. A. Chaturvedi, A. K. Sanyal, and N. H. McClamroch, "Rigid-body attitude control," IEEE Control Systems, vol. 31, no. 3, pp. 30-51, June 2011.

[3] J. T. Y. Wen and K. Kreutz-Delgado, "The attitude control problem," IEEE Transactions on Automatic Control, vol. 36, no. 10, pp. 11481162, Oct 1991.

[4] M. D. Shusters and D. Oh, "Three-axis attitude determination from vector observations," Journal of Guidance, Control, and Dynamics, vol. 4, no. 1, pp. 70-77, 2017/02/14 1981. [Online]. Available: http://dx.doi.org/10.2514/3.19717

[5] J. L. Crassidis, F. L. Markley, and Y. Cheng, "Survey of nonlinear attitude estimation methods," Journal of Guidance, Control, and Dynamics, vol. 30, no. 1, pp. 12-28, 2017/02/14 2007. [Online]. Available: http://dx.doi.org/10.2514/1.22452

[6] R. Mahony, T. Hamel, and J. M. Pflimlin, "Nonlinear complementary filters on the special orthogonal group," IEEE Transactions on Automatic Control, vol. 53, no. 5, pp. 1203-1218, June 2008.

$$
\begin{aligned}
& A(t)=\left[\begin{array}{cccccc}
0 & 0 & 0 & \frac{1}{2} R_{d} & \frac{1}{2} R_{d} & 0 \\
0 & \lambda_{\rho} & 0 & 0 & -\frac{1}{2} v_{\rho}^{T} \hat{R} & 0 \\
0 & 0 & \lambda_{\rho} I_{2}-W_{\rho}^{\perp} & 0 & \frac{1}{2} S\left(v_{\rho}\right) \hat{R} & 0 \\
-2 I_{b}^{-1} R_{d}^{T} W_{\gamma} & 0 & 0 & -\alpha I_{b}^{-1} & 0 & -I_{b}^{-1} G_{d} \\
-2 \Gamma_{1} R_{d}^{T} W_{\gamma} & -2 \lambda_{\rho} \Gamma_{1} \hat{R}^{T} v_{\rho} & 2 \Gamma_{1} \hat{R}^{T} S\left(v_{\rho}\right)\left(\lambda_{\rho} I_{2}-W_{\rho}^{\perp}\right) & 0 & 0 & 0 \\
0 & 0 & 0 & \Gamma_{2} G_{d}^{T} & 0 & 0
\end{array}\right], \\
& F\left(t, Z_{r e d}\right)=\left(\begin{array}{c}
\frac{1}{2}\left[\left(Z_{\tilde{q} 0} I d_{3}+S\left(Z_{\tilde{q}}\right)\right) R_{d}\left(Z_{\bar{\omega}}+Z_{\tilde{\theta}}\right)\right] \\
Z_{\bar{q}_{0}}\left(2 \lambda_{\rho} v_{\rho}^{T} Z_{\bar{q}}+Z_{\bar{q}}^{T} W_{\rho} Z_{\bar{q}}\right)-\frac{1}{2} Z_{\bar{q}}^{T} \hat{R} Z_{\tilde{\theta}} \\
\left.\lambda_{\rho} v_{\rho}^{T} Z_{\bar{q}} Z_{\bar{q}}^{\perp}+\frac{1}{2}\left[\left(Z_{\bar{q}_{0}} I d_{3}+S\left(Z_{\bar{q}}\right)\right) \hat{R} Z_{\tilde{\theta}}\right)\right]^{\perp} \\
-2 I_{b}^{-1} R_{d}^{T}\left(Z_{\tilde{q} 0} I d_{3}-S\left(Z_{\tilde{q}}\right)\right) W_{\gamma} Z_{\tilde{q}}-I_{b}^{-1} G\left(Z_{\bar{\omega}}+Z_{\tilde{\theta}}, \Gamma_{1}\left(Z_{\gamma}+Z_{\rho}\right)\right) Z_{\tilde{\Theta}} \\
\left.-2 \Gamma_{1} R_{d}^{T}\left[Z_{\tilde{q}_{0}} I d_{3}-S\left(Z_{\tilde{q}}\right)\right) W_{\gamma} Z_{\tilde{q}}\right]-2 \Gamma_{1} \hat{R}^{T}\left[\left(Z_{\bar{q}_{0}} I_{3}-S\left(Z_{\bar{q}}\right)\right) W_{\rho} Z_{\bar{q}}\right] \\
\Gamma_{2} G^{T}\left(Z_{\bar{\omega}}+Z_{\tilde{\theta}}, \Gamma_{1}\left(Z_{\gamma}+Z_{\rho}\right)\right) Z_{\bar{\omega}}
\end{array}\right) .
\end{aligned}
$$

7] J. Thienel and R. M. Sanner, "A coupled nonlinear spacecraft attitude controller and observer with an unknown constant gyro bias and gyro noise," IEEE Transactions on Automatic Control, vol. 48, no. 11, pp. 2011-2015, Nov 2003.

[8] P. Pounds, T. Hamel, and R. Mahony, "Attitude control of rigid body dynamics from biased imu measurements," in 2007 46th IEEE Conference on Decision and Control, Dec 2007, pp. 4620-4625.

[9] W. Luo, Y.-C. Chu, and K.-V. Ling, "Inverse optimal adaptive control for attitude tracking of spacecraft," IEEE Transactions on Automatic Control, vol. 50, no. 11, pp. 1639-1654, Nov 2005.

[10] L. Benziane, A. Benallegue, Y. Chitour, and A. Tayebi, "Velocity-free attitude stabilization with inertial vector measurements," Int. J. Robust. Nonlinear Control, vol. 26, pp. 2478-2493, 2016.

[11] A. Khosravian and M. Namvar, "Rigid body attitude control using a single vector measurement and gyro," IEEE Transactions on Automatic Control, vol. 57, no. 5, pp. 1273-1279, May 2012.

[12] T. Mercker and M. Akella, "Rigid-body attitude tracking with vector measurements and unknown gyro bias," Journal of Guidance, Control, and Dynamics, vol. 34, no. 5, pp. 1474-1484, 2017/02/14 2011. [Online]. Available: http://dx.doi.org/10.2514/1.53111

[13] J. Ahmed, V. Coppola, and D. Bernstein, "Adaptive asymptotic tracking of spacecraft attitude motion with inertia matrix identification," Journal of Guidance, Control, and Dynamics, vol. 21, no. 5, pp. 684-691, 2017/02/14 1998. [Online]. Available: http://dx.doi.org/10.2514/2.4310

[14] A. H. J. de Ruiter, "Observer-based adaptive spacecraft attitude control with guaranteed performance bounds," IEEE Transactions on Automatic Control, vol. 61, no. 10, pp. 3146-3151, Oct 2016.

[15] Z. Cai, M. S. de Queiroz, and D. M. Dawson, "A sufficiently smooth projection operator," IEEE Transactions on Automatic Control, vol. 51, no. 1, pp. 135-139, Jan 2006.

[16] A. Tayebi, A. Roberts, and A. Benallegue, "Inertial vector measurements based velocity-free attitude stabilization," IEEE Transactions on Automatic Control, vol. 58, no. 11, pp. 2893-2898, Nov 2013.

\section{APPENDIX}

The expressions of $A(t)$ and $F\left(t, Z_{\text {red }}\right)$ are given by 